Georgian Mathematical Journal

Volume 14 (2007), Number 1, 135-143

\title{
ON THE MEASURABILITY OF ADDITIVE FUNCTIONALS
}

\author{
ALEXANDER KHARAZISHVILI
}

Dedicated to the Memory of Professor Gaetano Fichera

\begin{abstract}
For an infinite-dimensional separable Hilbert space $H$, the problem of measurability of additive functionals $f: H \rightarrow \mathbf{R}$ with respect to various extensions of $\sigma$-finite diffused Borel measures on $H$ is discussed. It is shown that there exists an everywhere discontinuous additive functional $f$ on $H$ such that, for any $\sigma$-finite diffused Borel measure $\mu$ on $H$, this $f$ can be made measurable with respect to an appropriate extension of $\mu$. Special consideration is given to the case where $\mu$ is invariant or quasiinvariant under a subgroup of $H$.
\end{abstract}

2000 Mathematics Subject Classification: 28A05, 28D05.

Key words and phrases: Hilbert space, additive functional, Borel measure, extension of measure, Bernstein set.

Let $E$ be a topological space such that all singletons in $E$ are Borel subsets of $E$.

Recall that a Borel measure $\mu$ on $E$ is diffused (or continuous) if $\mu(\{x\})=0$ for each point $x \in E$.

We denote by the symbol $M(E)$ the class of all completions of $\sigma$-finite Borel measures on $E$. A set $X \subset E$ is called universally measurable with respect to $M(E)$ if, for any measure $\mu \in M(E)$, we have $X \in \operatorname{dom}(\mu)$ (cf. [1]). It is well known that if $E$ is a Polish space, then all analytic (also, co-analytic) subsets of $E$ are universally measurable with respect to $M(E)$ (for the proof, see, e.g., [1] or [2]). The family of all universally measurable sets in $E$ forms a $\sigma$-algebra of subsets of $E$.

A functional $f: E \rightarrow \mathbf{R}$ is called universally measurable if $f$ is measurable with respect to any $\mu \in M(E)$.

Let $H$ denote an infinite-dimensional separable Hilbert space and let $f$ : $H \rightarrow \mathbf{R}$ be a universally measurable additive functional. Then $f$ turns out to be continuous (see [3]; a more general result is presented in [4]). In fact, $f$ is continuous whenever it is universally measurable with respect to the class of the completions of all probability diffused Borel measures on $H$.

To demonstrate this, take such an $f$ and suppose to the contrary that $f$ is not continuous. Then, for every natural number $n$, there exists an element $h_{n} \in H$ satisfying the relations

$$
\left\|h_{n}\right\|=1, \quad\left|f\left(h_{n}\right)\right|>n \cdot 4^{n} .
$$


Let us put $e_{n}=h_{n} / 4^{n}$. Obviously, we have

$$
\left\|e_{n}\right\|=1 / 4^{n}, \quad\left|f\left(e_{n}\right)\right|>n \quad(n \in \omega) .
$$

Further, consider the Cantor space $C=\{0,1\}^{\omega}$ as a compact commutative group (with respect to the addition operation modulo 2) and equip $C$ with the Haar probability measure $\lambda$ which is actually isomorphic to the standard Lebesgue measure on the segment $[0,1]$.

Define a mapping $\psi: C \rightarrow H$ by the formula

$$
\psi\left(1_{A}\right)=\sum_{n \in A} e_{n} \quad(A \subset \omega),
$$

where $1_{A}$ denotes the characteristic function of a set $A \subset \omega$. It can easily be verified that:

(i) $\psi$ is continuous;

(ii) $\psi$ is injective;

(iii) $\left|(f \circ \psi)\left(1_{\{n\}}\right)\right|=\left|f\left(e_{n}\right)\right|>n$ for each $n<\omega$.

Let $\mu$ denote the distribution in $H$ of the random variable $\psi$. Clearly, $\mu$ is a Borel diffused probability measure on $H$ and, according to our assumption, $f$ is measurable with respect to the completion of $\mu$. This implies that the composition $f \circ \psi$ is measurable with respect to $\lambda$. Since this composition is also additive on the power-set $\mathcal{P}(\omega)$, we conclude (cf. [3], [4]) that the set

$$
\left\{(f \circ \psi)\left(1_{\{n\}}\right): n<\omega\right\}
$$

must be bounded in $H$, which is impossible in view of relation (iii). The contradiction obtained yields the required result.

Obviously, the same argument works for an arbitrary infinite-dimensional Banach space $E$ instead of $H$.

We thus see that only continuous linear functionals on $H$ can be universally measurable with respect to the class of all completions of $\sigma$-finite (equivalently, probability) diffused Borel measures on $H$.

For a finite-dimensional Euclidean space $\mathbf{R}^{n}$, we have a much stronger result: if an additive functional $f: \mathbf{R}^{n} \rightarrow \mathbf{R}$ is measurable with respect to the standard Lebesgue measure on $\mathbf{R}^{n}$, then $f$ is continuous (see, e.g., [5]). An analogous result is valid for any homomorphism $f$ acting from a $\sigma$-compact locally compact topological group $\Gamma$ into the additive group $\mathbf{R}$ and measurable with respect to the completion of the Haar measure on $\Gamma$ (here the so-called Steinhaus property of the Haar measure plays a significant role).

In this paper we introduce a different notion of universal measurability of functionals. It will be shown in the sequel that there exist universally measurable additive functionals on $H$ (in the sense of our notion) which are everywhere discontinuous. Then we will demonstrate how such universally measurable additive functionals can be applied to the measure extension problem.

Let $E$ be an arbitrary nonempty set and let $M$ be a class of measures on $E$ (we do not assume, in general, that the measures from $M$ are defined on the same $\sigma$-algebra of subsets of $E$ ). 
We will say that a functional $f: E \rightarrow \mathbf{R}$ is universally measurable with respect to $M$ if, for each measure $\mu \in M$, there exists a measure $\mu^{\prime}$ on $E$ extending $\mu$ and such that $f$ becomes measurable with respect to $\mu^{\prime}$.

Evidently, this definition is more general than the one given in the beginning of the paper. Also, in this definition we may replace the real line $\mathbf{R}$ by an uncountable Polish space or, more generally, by an uncountable Borel subset of a Polish space (since, according to the well-known result of descriptive set theory, any two uncountable Borel subsets of a Polish space are Borel isomorphic).

Recall that a subset $X$ of a topological space $E$ is a Bernstein set in $E$ if, for each nonempty perfect set $P \subset E$, we have $P \cap X \neq \varnothing$ and $P \cap(E \backslash X) \neq \varnothing$. Various properties of Bernstein sets are discussed in [2], [5], [6] and [7]. In particular, any uncountable Polish space contains a Bernstein subset and the cardinality of such a subset is equal to the cardinality of the continuum (denoted by $\mathbf{c})$.

For our further purposes, we need two lemmas.

Lemma 1. The Hilbert space $H$ can be represented in the form

$$
H=X_{1}+X_{2} \quad\left(X_{1} \cap X_{2}=\{0\}\right),
$$

where $X_{1}$ and $X_{2}$ are Bernstein subsets of $H$ and, simultaneously, they are vector spaces over the field $\mathbf{Q}$ of all rationals.

Proof. The argument is fairly standard (cf. [10], p. 25, Theorem 5). Denote by $\alpha$ the least ordinal number of cardinality continuum and let $\left\{P_{\xi}: \xi<\alpha\right\}$ be an enumeration of all nonempty perfect subsets of $H$. By using the method of transfinite recursion it is not difficult to construct two $\alpha$-sequences

$$
\left\{x_{\xi}: \xi<\alpha\right\} \subset H, \quad\left\{y_{\xi}: \xi<\alpha\right\} \subset H
$$

satisfying the following conditions:

(a) the family $\left\{x_{\xi}: \xi<\alpha\right\} \cup\left\{y_{\xi}: \xi<\alpha\right\}$ is linearly independent over Q;

(b) for any ordinal $\xi<\alpha$, we have $x_{\xi} \in P_{\xi}$ and $y_{\xi} \in P_{\xi}$.

Now, let us take as $X_{1}$ the vector space (over $\mathbf{Q}$ ) generated by $\left\{x_{\xi}: \xi<\alpha\right\}$. Further, let $X_{2}$ denote a maximal vector subspace $V$ of $H$ (over $\mathbf{Q}$ ) such that

$$
V \cap X_{1}=\{0\}, \quad\left\{y_{\xi}: \xi<\alpha\right\} \subset V .
$$

The existence of $V$ is evident and it is also easy to see that $X_{1}$ and $X_{2}$ are the required Bernstein subsets of $H$.

Remark 1. The proof given above works for an arbitrary Banach space of cardinality continuum, not necessarily separable (cf. again [10], p. 25, Theorem 5).

Lemma 2. There exists an additive functional $f: H \rightarrow \mathbf{R}$ having the following property: for any $\sigma$-finite diffused Borel measure $\mu$ on $H$ and for any $\sigma$-finite measure $\nu$ on $\mathbf{R}$, the graph of $f$ is a $(\mu \times \nu)$-thick subset of the product space $H \times \mathbf{R}$ (i.e. this graph intersects every $(\mu \times \nu)$-measurable set of strictly positive measure). 
Proof. First, applying Lemma 1, we represent $H$ in the form

$$
H=X_{1}+X_{2} \quad\left(X_{1} \cap X_{2}=\{0\}\right),
$$

where $X_{1}$ and $X_{2}$ are Bernstein subsets of $H$ and, simultaneously, they are vector spaces over $\mathbf{Q}$. Observe now that, in view of the equalities

$$
\operatorname{card}\left(X_{1}\right)=\mathbf{c}, \quad \operatorname{card}(\mathbf{R})=\mathbf{c},
$$

the vector spaces (over $\mathbf{Q}$ ) $X_{1}$ and $\mathbf{R}$ are isomorphic to each other. Let

$$
\phi: X_{1} \rightarrow \mathbf{R}
$$

denote some isomorphism between these two spaces. We define a functional $f$ as follows. Take any $x \in H$. This $x$ admits a unique representation in the form $x=x_{1}+x_{2}$, where $x_{1} \in X_{1}$ and $x_{2} \in X_{2}$. Let us put

$$
f(x)=f\left(x_{1}+x_{2}\right)=\phi\left(x_{1}\right) .
$$

Obviously, $f$ is an additive functional on $H$. Further, fix a $\sigma$-finite diffused Borel measure $\mu$ on $H$ and a $\sigma$-finite measure $\nu$ on $\mathbf{R}$. We assert that the graph of $f$ is $(\mu \times \nu)$-thick in $H \times \mathbf{R}$. Indeed, if $Z$ is an arbitrary $(\mu \times \nu)$-measurable set with $(\mu \times \nu)(Z)>0$, then, according to the Fubini theorem, there exists a point $t \in \mathbf{R}$ such that $\mu(Z(t))>0$, where

$$
Z(t)=\{x \in H:(x, t) \in Z\} .
$$

Consider the point $x_{1}=\phi^{-1}(t)$. Since $X_{2}$ is a Bernstein subset of $H$ and $Z(t)$ is an uncountable Borel subset of $H$, we have

$$
X_{2} \cap\left(Z(t)-x_{1}\right) \neq \varnothing .
$$

Choose a point $x_{2}$ from the set $X_{2} \cap\left(Z(t)-x_{1}\right)$ and define $x=x_{1}+x_{2}$. Then we get

$$
x=x_{1}+x_{2} \in Z(t), \quad(x, t) \in Z, \quad(x, f(x))=(x, t), \quad(x, f(x)) \in Z,
$$

which completes the proof of Lemma 2.

Let $f: H \rightarrow \mathbf{R}$ be as in Lemma 2 . Note that such an $f$ is everywhere discontinuous on $H$ because the graph of any measurable (in particular, continuous) functional is always of measure zero, so it cannot be thick in the product space $H \times \mathbf{R}$. Fix a Borel diffused probability measure $\lambda$ on $\mathbf{R}$. For instance, we may take as $\lambda$ an arbitrary Borel probability measure equivalent to the standard Lebesgue measure on $\mathbf{R}$ (assuming that the latter measure is restricted to the Borel $\sigma$-algebra of $\mathbf{R}$ ). Now, let $\mu$ be any $\sigma$-finite diffused Borel measure on $H$. For each $(\mu \times \lambda)$-measurable set $Z \subset H \times \mathbf{R}$, we denote

$$
Z^{\prime}=\{x \in H:(x, f(x)) \in Z\} .
$$

Further, we put

$$
\mathcal{S}^{\prime}=\left\{Z^{\prime}: Z \in \operatorname{dom}(\mu \times \lambda)\right\} .
$$

It can easily be verified that $\mathcal{S}^{\prime}$ is a $\sigma$-algebra of subsets of $H$ containing the Borel $\sigma$-algebra of $H$. Finally, we define a functional $\mu^{\prime}$ on $\mathcal{S}^{\prime}$ by putting

$$
\mu^{\prime}\left(Z^{\prime}\right)=(\mu \times \lambda)(Z) \quad(Z \in \operatorname{dom}(\mu \times \lambda)) .
$$


A straightforward verification shows that the definition of $\mu^{\prime}$ is correct (in view of the $(\mu \times \lambda)$-thickness of the graph of $f)$. Also, $\mu^{\prime}$ turns out to be a measure on $\mathcal{S}^{\prime}$ which extends the original measure $\mu$. We thus come to the following statement.

Theorem 1. The additive functional $f: H \rightarrow \mathbf{R}$ is universally measurable (in the above-mentioned sense) with respect to the class of all $\sigma$-finite diffused Borel measures on $H$.

Proof. It suffices to show that, for any Borel diffused $\sigma$-finite measure $\mu$ on $H$, the functional $f$ is measurable with respect to the measure $\mu^{\prime}$. For this purpose, take an arbitrary Borel subset $B$ of $\mathbf{R}$. Clearly, we may write

$$
f^{-1}(B)=\{x \in H: f(x) \in B\}=\{x \in H:(x, f(x)) \in H \times B\} \in \mathcal{S}^{\prime},
$$

which establishes the measurability of $f$ with respect to $\mu^{\prime}$ and, therefore, finishes the proof.

Let us indicate some other properties of $f$ useful from the measure-theoretical point of view. It is well known that there exists no nonzero $\sigma$-finite Borel measure on $H$ invariant (quasiinvariant) under the group of all translations of $H$ (see, for instance, [8]). At the same time, there exist various nonzero $\sigma$ finite Borel measures on $H$ which are invariant under everywhere dense vector subspaces of $H$ (see [9]). Let $\mu$ be any such measure on $H$. We already know that $f$ is measurable with respect to $\mu^{\prime}$. So it is natural to ask whether the measure $\mu^{\prime}$ remains invariant under the same everywhere dense vector subspace of $H$. It turns out that the answer to this question is always positive.

In order to demonstrate this, let us slightly change the construction presented above (cf. [11]). We replace the real line $\mathbf{R}$ by the one-dimensional unit torus $\mathbf{T}$ regarded as a compact commutative group. The torus $\mathbf{T}$ is equipped with the Haar probability measure (which, actually, coincides with the Lebesgue probability measure on $\mathbf{T}$ invariant under all translations of $\mathbf{T})$. We denote the latter measure by the same symbol $\lambda$.

The following statement is true.

Lemma 3. There exists a group homomorphism $f: H \rightarrow \mathbf{T}$ having the property that, for any Borel $\sigma$-finite diffused measure $\mu$ on $H$, the graph of $f$ is $a(\mu \times \lambda)$-thick subset of the product space $H \times \mathbf{T}$.

Proof. The argument is very similar to that used in the proof of Lemma 2. Again, let us represent $H$ in the form

$$
H=X_{1}+X_{2} \quad\left(X_{1} \cap X_{2}=\{0\}\right),
$$

where both $X_{1}$ and $X_{2}$ are Bernstein subsets of $H$ and, simultaneously, they are vector spaces over the field $\mathbf{Q}$ of all rationals. Taking into account the fact that $X_{1}$ and $\mathbf{R}$ are isomorphic as vector spaces over $\mathbf{Q}$, it is not difficult to define a surjective group homomorphism

$$
\phi: X_{1} \rightarrow \mathbf{T}
$$


Now, if $x$ is an arbitrary element of $H$, then $x$ admits a unique representation in the form

$$
x=x_{1}+x_{2} \quad\left(x_{1} \in X_{1}, \quad x_{2} \in X_{2}\right) .
$$

So, we may define

$$
f(x)=f\left(x_{1}+x_{2}\right)=\phi\left(x_{1}\right) .
$$

In this way, we obtain a group homomorphism $f: H \rightarrow \mathbf{T}$. Let $\mu$ be an arbitrary $\sigma$-finite diffused Borel measure on $H$. Take any set $Z \in \operatorname{dom}(\mu \times \lambda)$ with $(\mu \times \lambda)(Z)>0$. According to the Fubini theorem, $\mu(Z(t))>0$ for some $t \in \mathbf{T}$. Since $\phi$ is a surjection, we can find $x_{1} \in X_{1}$ such that $\phi\left(x_{1}\right)=t$. Keeping in mind that $X_{2}$ is a Bernstein subset of $H$, we get

$$
X_{2} \cap\left(Z(t)-x_{1}\right) \neq \varnothing .
$$

Choose an element $x_{2}$ from the set $X_{2} \cap\left(Z(t)-x_{1}\right)$ and put $x=x_{1}+x_{2}$. As in the proof of Lemma 2, we easily conclude that $(x, f(x)) \in Z$, which shows the $(\mu \times \lambda)$-thickness of the graph of $f$ in the product space $H \times \mathbf{T}$.

By using the homomorphism $f: H \rightarrow \mathbf{T}$ described in the preceding lemma, we can extend any $\sigma$-finite diffused Borel measure $\mu$ given on $H$ to the measure $\mu^{\prime}$. The scheme of obtaining the extension $\mu^{\prime}$ of $\mu$ is the same as before.

Theorem 2. Let $\mu$ be a $\sigma$-finite Borel measure on $H$ invariant (quasiinvariant) under some subgroup $G$ of $H$. Then the measure $\mu^{\prime}$ is also invariant (quasiinvariant) under $G$.

Proof. It suffices to consider the case of an invariant measure $\mu$ (for quasiinvariant measures the argument is absolutely analogous). Take any set $Z^{\prime}$ from the domain of $\mu^{\prime}$. According to the definition, we may write

$$
Z^{\prime}=\{x \in H:(x, f(x)) \in Z\},
$$

where $Z$ belongs to the domain of $\mu \times \lambda$. For each $g \in G$, we have $Z^{\prime}+g=\{x \in H:(x-g, f(x-g)) \in Z\}=\{x \in H:(x, f(x)) \in Z+(g, f(g))\}$. Since the product measure $\mu \times \lambda$ is $(G \times \mathbf{T})$-invariant, we may write

$$
(\mu \times \lambda)(Z)=(\mu \times \lambda)(Z+(g, f(g)))
$$

and, consequently, $\mu^{\prime}\left(Z^{\prime}\right)=\mu^{\prime}\left(Z^{\prime}+g\right)$. This completes the proof of the theorem.

Obviously, for any nonzero $\sigma$-finite Borel measure $\mu$ on $H$, the measure $\mu^{\prime}$ obtained by using the group homomorphism $f: H \rightarrow \mathbf{T}$ is a proper extension of $\mu$. But the $\sigma$-algebra $\operatorname{dom}\left(\mu^{\prime}\right)$ is not significantly larger than the $\sigma$-algebra $\operatorname{dom}(\mu)$. Indeed, it can easily be seen that $\operatorname{dom}\left(\mu^{\prime}\right)$ is a countably generated $\sigma$-algebra and therefore the measure $\mu^{\prime}$ is separable. If we wish to extend $\mu$ to a nonseparable measure, then we must replace the torus $\mathbf{T}$ by the infinitedimensional torus $\mathbf{T}^{\mathbf{c}}$ which is the topological product of continuumly many copies of $\mathbf{T}$. Let $\nu$ denote the Haar probability measure on $\mathbf{T}^{\mathbf{c}}$. This measure is nonseparable and has the following property: there exists a family of sets

$$
\left\{Z_{i}: i \in I\right\} \subset \operatorname{dom}(\nu)
$$


such that:

(1) $\operatorname{card}(I)=\mathbf{c}$;

(2) $(\forall i \in I)\left(\nu\left(Z_{i}\right)>0\right)$;

(3) for every $\nu$-measurable set $Z$ with $\nu(Z)>0$, there is a set $Z_{i}$ from this family contained in $Z$.

The proof of the existence of $\left\{Z_{i}: i \in I\right\}$ with the above-mentioned property can be found, e.g., in [12].

Lemma 4. There exists a group homomorphism $f: H \rightarrow \mathbf{T}^{\mathbf{c}}$ such that, for any $\sigma$-finite diffused Borel measure $\mu$ on $H$, the graph of $f$ is a $(\mu \times \nu)$-thick subset of $\mathbf{R} \times \mathbf{T}^{\mathbf{c}}$.

Proof. Fix a family of sets $\left\{Z_{i}: i \in I\right\} \subset \operatorname{dom}(\nu)$ satisfying the relations (1) (3) given above. Without loss of generality, we may suppose that the set $I$ of indices is well-ordered and its order type coincides with the least ordinal number $\alpha$ whose cardinality is equal to $\mathbf{c}$. In other words, we may put

$$
\left\{Z_{i}: i \in I\right\}=\left\{Z_{\xi}: \xi<\alpha\right\} .
$$

As before, we represent our Hilbert space $H$ in the form

$$
H=X_{1}+X_{2} \quad\left(X_{1} \cap X_{2}=\{0\}\right),
$$

where $X_{1}$ and $X_{2}$ are some Bernstein subsets of $H$ and, simultaneously, they are vector spaces over $\mathbf{Q}$. Now, it is not difficult to construct two injective $\alpha$-sequences

$$
\left\{y_{\xi}: \xi<\alpha\right\} \subset X_{1}, \quad\left\{z_{\xi}: \xi<\alpha\right\} \subset \mathbf{T}^{\mathbf{c}}
$$

satisfying the following conditions:

(a) the family $\left\{y_{\xi}: \xi<\alpha\right\}$ is linearly independent over $\mathbf{Q}$;

(b) for each $\xi<\alpha$, we have $z_{\xi} \in Z_{\xi}$.

Further, we put

$$
\phi\left(y_{\xi}\right)=z_{\xi} \quad(\xi<\alpha)
$$

Taking into account the condition (a) and the fact that $\mathbf{T}^{\mathbf{c}}$ is a divisible group, we can extend the mapping $\phi$ to a group homomorphism

$$
\phi: X_{1} \rightarrow \mathbf{T}^{\mathbf{c}} .
$$

In view of (b), the set $\phi\left(X_{1}\right)$ is $\nu$-thick in $\mathbf{T}^{\mathbf{c}}$.

Now, take any $x \in H$. We have a unique representation $x=x_{1}+x_{2}$, where $x_{1} \in X_{1}$ and $x_{2} \in X_{2}$. Define

$$
f(x)=f\left(x_{1}+x_{2}\right)=\phi\left(x_{1}\right) .
$$

In this manner, we get a group homomorphism

$$
f: H \rightarrow \mathbf{T}^{\mathbf{c}} .
$$

Let us show that $f$ is the required one. Consider any $\sigma$-finite diffused Borel measure $\mu$ on $H$. Let $Z$ be an arbitrary $(\mu \times \nu)$-measurable set with $(\mu \times \nu)(Z)>$ 0 . Applying again the Fubini theorem and the $\nu$-thickness of $\phi\left(X_{1}\right)$ in $\mathbf{T}^{\mathbf{c}}$, we 
see that $\mu(Z(t))>0$ for some $t \in \phi\left(X_{1}\right)$. Choose an element $x_{1} \in X_{1}$ such that $\phi\left(x_{1}\right)=t$. Since $X_{2}$ is a Bernstein set in $H$, we have

$$
X_{2} \cap\left(Z(t)-x_{1}\right) \neq \varnothing .
$$

Consequently, for some $x_{2} \in X_{2} \cap\left(Z(t)-x_{1}\right)$, we may put $x=x_{1}+x_{2}$. Then an easy verification shows that $(x, f(x)) \in Z$ (cf. the final part of the proof of Lemma 2).

Thus, the graph of $f$ is $(\mu \times \nu)$-thick in the product space $H \times \mathbf{T}^{\mathbf{c}}$ and $f$ is universally measurable (in our sense) with respect to the class of all $\sigma$-finite diffused Borel measures on $H$.

Applying the previous lemma, we readily come to the following result.

Theorem 3. Let a group homomorphism $f: H \rightarrow \mathbf{T}^{\mathbf{c}}$ be as in Lemma 4 . For every $\sigma$-finite diffused Borel measure $\mu$, denote by $\mu^{\prime}$ the extension of $\mu$ obtained by using this group homomorphism. Then one can assert that:

1) $\mu^{\prime}$ is a nonseparable measure;

2) if $\mu$ is invariant (quasiinvariant) under some group $G \subset H$, then $\mu^{\prime}$ is also invariant (quasiinvariant) under $G$.

Remark 2. Analogous results can be obtained for the case of the $n$-dimensional Euclidean space $\mathbf{R}^{n}(n \geq 1)$ instead of $H$. The argument and technique remain the same. In particular, we may assert that there exists an additive functional $f: \mathbf{R}^{n} \rightarrow \mathbf{R}$ which is discontinuous at all points of $\mathbf{R}^{n}$ and is universally measurable (in our sense) with respect to the class of all $\sigma$-finite diffused Borel measures on $\mathbf{R}^{n}$.

Remark 3. It should be noted that if a vector space $E$ (over the field $\mathbf{Q}$ ) is of cardinality continuum, then, assuming Martin's Axiom, there exists an injective additive functional $f: E \rightarrow \mathbf{R}$ which is absolutely nonmeasurable with respect to the class of all nonzero $\sigma$-finite diffused measures on $E$. Indeed, the existence of such an $f$ is implied by the fact that there exists a generalized Luzin subset of $\mathbf{R}$ being simultaneously a vector space over $\mathbf{Q}$ (for more details, see, e.g., $[13])$.

Remark 4. As already has been mentioned, the standard notion of universal measurability of functionals on a Hilbert space yields nothing new for the class of additive functionals because every universally measurable additive functional turns out to be continuous. Moreover, it was shown in [14] that even the midpoint convexity of a given functional with its universal measurability (in the standard sense) implies its continuity. This result can be regarded as an analog of Sierpinski's old theorem stating that any Lebesgue measurable mid-point convex function defined on a finite-dimensional Euclidean space is necessarily continuous (see, for instance, [5]).

In this context, the paper [15] should also be pointed out, where the universal measurability (in the standard sense) with respect to the class of all Gaussian measures on a Hilbert space is considered. 


\section{REFERENCES}

1. N. Bourbaki, Elements of mathematics. Integration. (Russian) Nauka, Moscow, 1977.

2. K. Kuratowski, Topology. Vol. I. (Translated from the French) Academic Press, New York-London; Panstwowe Wydawnictwo Naukowe, Warsaw, 1966.

3. I. LABUdA and R. D. MAUldin, Problem 24 of the "Scottish book" concerning additive functionals. Colloq. Math. 48(1984), No. 1, 89-91.

4. I. LabudA, Universal measurability and summable families in tvs. Nederl. Akad. Wetensch. Indag. Math. 41(1979), No. 1, 27-34.

5. M. Kuczma, An introduction to the theory of functional equations and inequalities. Cauchy's equation and Jensen's inequality. Scientific Publications of the University of Silesia, 489. Państwowe Wydawnictwo Naukowe (PWN), Warsaw, 1985.

6. J. C. Oxтовy, Measure and category. A survey of the analogies between topological and measure spaces. Graduate Texts in Mathematics, Vol. 2. Springer-Verlag, New YorkBerlin, 1971.

7. J. C. Morgan, II, Point set theory. Monographs and Textbooks in Pure and Applied Mathematics, 131. Marcel Dekker, Inc., New York, 1990.

8. A. V. Skorokhod, Integration in Hilbert space. (Translation from Russian) Ergebnisse der Mathematik und ihrer Grenzgebiete. Band 79.Springer-Verlag, Berlin-Heidelberg-New York, 1974.

9. A. B. Kharazishvili, Invariant measures in Hilbert space. (Russian) Soobshch. Akad. Nauk Gruzin. SSR 114(1984), No. 1, 45-48.

10. A. B. Kharazishvili, Nonmeasurable sets and functions. North-Holland Mathematics Studies, 195. Elsevier Science B.V., Amsterdam, 2004.

11. K. Kodaira and S. KaKUTANi, A non-separable translation invariant extension of the Lebesgue measure space. Ann. of Math. (2) 52(1950), 574-579.

12. E. Hewitt and K. A. Ross, Abstract harmonic analysis. Vol. I: Structure of topological groups. Integration theory, group representations. Die Grundlehren der mathematischen Wissenschaften, Bd. 115 Academic Press, Inc., Publishers, New York; Springer-Verlag, Berlin-Göttingen-Heidelberg, 1963, Berlin, 1963.

13. A. Kharazishvili and A. Kirtadze, On the measurability of functions with respect to certain classes of measures. Georgian Math. J. 11(2004), No. 3, 489-494.

14. K. Fischer and Z. SŁodkowski, Christensen zero sets and measurable convex functions. Proc. Amer. Math. Soc. 79(1980), No. 3, 449-453.

15. M. P. Kats, Continuity of universally measurable linear mappings. (Russian) Sibirsk. Mat. Zh. 23(1982), No. 3, 83-90.

(Received 5.04.2006)

Author's addresses:

A. Razmadze Mathematical Institute

1, M. Aleksidze St., Tbilisi 0193

Georgia

I. Chavchavadze State University

32, I. Chavchavadze St., Tbilisi 0128

Georgia

E-mail: kharaz2@yahoo.com 